\title{
Gluelump spectrum in the QCD string model
}

\author{
Yu.A.Simonov \\ State Research Center \\ Institute of Theoretical and Experimental Physics, \\ Moscow, Russia
}

\begin{abstract}
Spectrum of gluons in the adjoint source field is computed analytically using the QCD string Hamiltonian, containing only one parameter - string tension, fixed by meson and glueball spectrum. Spin splitting is shown to be small. A good agreement is observed with spacially generated gluelump states measured on the lattice. Important role of gluelumps defining the behaviour of field-strength correlators is stressed and correspondence with earlier computations of the latter is established.
\end{abstract}

\section{Introduction}

Gluelumps [1, 2] are not physical objects and their spectrum cannot be measured on experiment. However, as will be discussed below, they play more fundamental role than any other hadrons since gluelump masses define field correlators in the QCD vacuum and in particular string tension. The purpose of the present study is to calculate analytically the gluelamp spectrum in the framework of the so-called QCD string model (QCDSM). This model was developed in the earlier papers [ [0, 团] for spinless quarks, and was augmented by the spin-dependent terms treated as a perturbation [5, [6]. The resulting Hamiltonian of the rotating string with quarks or gluons at the ends was recently systematically applied to mesons, hybrids and glueballs (see review in [6] and recent developments in [7, 8]). Three features are characteristic for the model. First, QCDSM is directly derived from QCD with few assumptions supported by the lattice data, i.e. the minimal area law for the 
Wilson loop, and the dominant role of valence quarks and gluons. Second, it is fully relativistic and the Hamiltonian can be obtained in the c.m. system [4], or in the light-cone system [9] or else on any other hypersurface. Third, the model contains the minimal number of parameters: fundamental string tension $\sigma_{f}=0.18 \mathrm{GeV}^{2}$ defined by the meson Regge slope, the strong coupling constant $\alpha_{s}$ (taken as constant in first approximation, $\alpha_{s}(\mu)$, where $\mu$ is an inverse size of the system, or with the freezing behaviour $\alpha_{s}(r)$ [10] in more accurate calculations), and finally the quark (antiquark) selfenergy $C_{0}=-0.25 \mathrm{GeV}$, which should be subtracted for each quark from the mass of a meson, hybrid, or baryon. Note, that for gluons this term is absent because of gauge invariance and therefore spectrum of glueballs and gluelumps is defined only by $\sigma_{f}$ and $\alpha_{s}$.

It is remarkable, that with these fixed universal parameters one obtains spectrum of all hadronic systems in good agreement with lattice data and experiment [6]. In particular, the glueball spectrum depending only on $\sigma_{f}$ (with spin splittings depending also on $\alpha_{s}$ ) comes out [11 in remarkable agreement with recent lattice data [12].

At the same time the heavy-light mesons have been calculated in this method in [13] including quark decay constants $f_{M}, M=B, B_{s}, D, D_{s}$ again in good agreement with experiment and other approaches.

This gives a hope that the QCDSM can be used also for the system containing a valence gluon connected by an adjoint string to the adjoint source ("infinitely heavy gluon") - the so-called gluelump [1, [2].

Gluelump has a resemblance both to glueballs and to heavy-light mesons. In the framework of the string model, gluelump is a glueball with the adjoint string between gluons when one gluon is made static, and one expects the same structure of the spectrum of the LS-coupling type, which is obtained in [11] and similar to the lattice spectrum calculated in [12].

On the other hand, gluelump differs (within the model) from heavylight mesons only in that the fundamental string is replaced by the adjoint one (plus minor differences in the spin splitting terms and the role of color Coulomb forces).

Therefore the calculation of the gluelump spectrum is expected to be as successful as in the case of glueballs and heavy-light mesons. From physical point of view the gluelumps are important at least in two respects. First of all gluelumps play a fundamental role in the nonperturbative structure of the QCD vacuum, since gluelump Green's functions are connected to the field correlators $\left\langle F_{\mu \nu}(x) F_{\lambda \sigma}(0)\right\rangle$ or its invariant parts $D(x), D_{1}(x)$, introduced in 
[14], which are basic elements in the Method of Field Correlators (MFC). In particular, the gluon correlation length $T_{g}$, defining the nonperturbative dynamics of confinement [15, [16] is simply the inverse mass of the lowest gluelump, and was computed in this way before in several systematic studies [17.

Secondly, gluelump masses define the screening length for the static potential in given representation $D$ of $S U(3)$, namely the minimal length of the string, which may decay into two gluelumps.

Recent precise measurements of static potentials $V_{D}(r)$ made in [18] revealed an accurate (within 1\%) Casimir scaling without any signs of screening (string breaking) at all distances $0.05 \mathrm{fm} \leq r \leq 1.2 \mathrm{fm}$. To understand these results one should know screening length for all $D$.

The structure of the paper is as follows. In the first part of the paper the gluelump spectrum is computed, with the corresponding Hamiltonian constructed in section 2, spin splittings considered in section 3, and spectrum calculation in section 4. Comparison to the lattice data and the bag model is given in section 5. Section 6 contains conclusions and outlook, while Appendix is devoted to the details of hyperspherical formalism used for calculation of two-gluon gluelumps.

\section{The gluelump Hamiltonian}

The starting point is the gluelump Green's function $G^{\text {glump }}(x, y)$ which is obtained from the initial and final gluelump operators $\Psi^{(i n, o u t)}$, expressed in terms of the valence gluon field $a_{\mu}$ and background gluon field $B_{\mu}$ so that the total field is $A_{\mu}=B_{\mu}+a_{\mu}$, with the gauge transformation properties

$$
B_{\mu} \rightarrow U^{+}\left(B_{\mu}+\frac{i}{g} \partial_{\mu}\right) U, \quad a_{\mu} \rightarrow U^{+} a_{\mu} U .
$$

Similarly to the glueball case one can write

$$
G^{g l u m p}(x, y)=\left\langle\operatorname{tr}_{a d j}\left(\Gamma^{(o u t)}(x) G_{\mu \nu}(x, y) \Gamma^{i n}(y) \Phi(y, x)\right)\right\rangle_{B},
$$

where $\Phi(x, y)$ is the parallel transporter representating the adjoint source Green's function:

$$
\Phi(x, y)=P \exp \left(i g \int_{y}^{x} \hat{B}_{\mu}(z) d z_{\mu}\right), \quad \hat{B}_{\mu}=B_{\mu}^{a} T^{a}
$$


and $G_{\mu \nu}(x, y)$ is the valence gluon Green's function [3]

$$
\begin{gathered}
G_{\mu \nu}(x, y)=\left(D^{2} \delta_{\mu \nu}+2 i g F_{\mu \nu}(B)\right)_{x, y}^{-1}= \\
=\int_{0}^{\infty} d s \int D z e^{-K} P \exp \left[i g \int_{y}^{x} B_{\mu} d z_{\mu}+2 g \int_{0}^{s} F_{\mu \nu}(z(\tau)) d \tau\right]
\end{gathered}
$$

In (2), $\Gamma^{\text {(out,in) }}(x)$ are operators defining quantum numbers of final and initial gluelump states, below in Table 1 listed are examples for the lowest mass states.

The last term in the exponent in (1) describes the interaction of the gluon spin with the background $B_{\mu}$ (and with the adjoint string, which will be created out of it). Here as well as in the glueball case [11] we shall treat this interaction as perturbation to be discussed in section 3, and disregard it in the first approximation. Then Eq. (目) reduces to the average of the adjoint Wilson loop with the contour $C$ consisting of the straight line of the source and the path $z(\tau)$ of the valence gluon, integrated upon in (4).

Assuming the area law for the adjoint Wilson loop in agreement with lattice measurements [18], one can go over in a standard way [3, 4] from the Green's function $G^{(\text {glump })}$ to the corresponding Hamiltonian $H^{(\text {glump })}$, connected by

$$
G^{\text {glump }}(x, y)=\left\langle\text { out }\left|\exp \left(-H^{(\text {glump })}|x-y|\right)\right| i n\right\rangle .
$$

The resulting Hamiltonian can be obtained from that of the $q \bar{q}$ system [4, 6] when mass of one quark is zero and mass of another going to infinity, while the quark string tension $\sigma_{f}$ is replaced by $\sigma_{a d j}=\frac{9}{4} \sigma_{f}$. One obtains

$$
\begin{gathered}
H^{\text {(glump })}=\frac{\mu}{2}+\frac{p_{r}^{2}}{2 \mu}+\frac{L(L+1) / r^{2}}{2\left(\mu+\int_{0}^{1} d \beta \beta^{2} \nu(\beta)\right)} \\
+\frac{\sigma_{a d j}^{2} r^{2}}{2} \int_{0}^{1} \frac{d \beta}{\nu(\beta)}+\int_{0}^{1} \frac{\nu(\beta)}{2} d \beta .
\end{gathered}
$$

Here $\mu$ and $\nu(\beta)$ are respectively the constituent gluon mass and energy density along the string coordinate $\beta$, to be found from minimization of the Hamiltonian.

For the angular momentum $L=0$ the stationary point in $\nu(\beta)$ yields a simple answer

$$
H^{(\text {glump })}(L=0)=\frac{\mu}{2}+\frac{p_{r}^{2}}{2 \mu}+\sigma_{a d j} r .
$$


For $L>0$ instead of solving for the complicated operator (6) we shall treat effect of $L$ perturbatively as in 4 , yielding accuracy around $5 \%$ for $L \leq 3$, namely

$$
H^{(\text {glump })}(L>0)=\frac{\mu}{2}+\frac{\mathbf{p}_{r}^{2}}{2 \mu}+\sigma_{a d j} r+\Delta M_{L}
$$

where we have defined as in [四]

$$
\Delta M_{L}=-\frac{4 L(L+1) \sigma_{a d j}^{2}}{3 M_{0}^{3}}
$$

and $\mu$ in $(8)$ is to be found from the minimum of the mass eigenvalues of $H^{\text {(glump) }}$, (accuracy of this procedure was found in [6, 19] to be better than $5 \%)$.

\section{Spin-dependent and color Coulomb inter- action}

We now take into account the term $F_{\mu \nu}$ in (丑), which yields the gluon spin $\mathbf{S}$ dependence, since

$$
-2 i F_{i k}=2 \mathbf{S B}, \quad i k=1,2,3 .
$$

The analysis of the spin-dependent terms can be done as in the case of glueballs [11] and heavy quarkonia [5] (note however, that nowhere we use the nonrelativistic inverse mass expansion, instead the lowest (Gaussian) field correlator $\langle F F\rangle$ is retained in the average of (雨), this procedure was tested in 20] to yield accuracy around 1\%).

As a result one obtains

$$
\Delta H_{L S}=\Delta H_{L S}(\text { Thomas })+\Delta H_{L S}(\text { pert })
$$

where

$$
\Delta H_{L S}(\text { Thomas })=-\sigma_{a d j} \frac{\mathbf{L S}}{2 \mu^{2}}\left\langle\frac{f(r)}{r}\right\rangle \equiv b^{(\text {Thomas })} \mathbf{L S}
$$

and $f(r=\infty)=1$ takes into account that confining interaction starts at small $r<T_{g}$, as $r^{2}$, which significantly decreases the matrix element $\left\langle\frac{f(r)}{r}\right\rangle$, see [5] for details, and [11] for numerical results for the glueball case.

With $\Delta H$ (pert) the situation is more subtle. Indeed the one-gluon exchange does not contribute to the potential $V_{1}(r)$ (we stick to the standard 
notations of Eichten-Feinberg-Gromes approach), and the latter is the only $L S$ piece surviving for the gluelump case. Hence one should consider the one-loop contribution, similar to the one considered in 21] for heavy quarkonia, in the limit of one mass infinite and another equal to $\mu$. In absence of actual calculations for the gluelump case, and to have an orientation, we shall translate the result of [21] replacing $C_{F} \rightarrow C_{A}$, and $m_{1} \rightarrow \mu, m_{2} \rightarrow \infty$. The result is

$$
\Delta H_{L S}(\text { pert })=\frac{\mathbf{L S}}{\mu^{2} r^{3}} \frac{\alpha_{s}^{2} C_{A}^{2}}{2 \pi}\left(2-\ln \mu r-\gamma_{E}\right) \equiv b^{(\text {pert })} \mathbf{L S} .
$$

As we shall see the overall magnitude of $\Delta H_{L S}$ and of its parts is small, and finally can be neglected. For an estimate we shall take the brackets in (13) to be equal to one, and the matrix element $\left\langle\frac{1}{r^{3}}\right\rangle$ can be found from the equality [11]

$$
L(L+1)\left\langle\frac{1}{r^{3}}\right\rangle=\mu \sigma_{a d j}
$$

Consider now the contribution of perturbative gluon exchanges to the interaction between the valence gluon and static source.

Here we adopt the same attitude that was suggested and discussed in detail in 11]. Namely, the perturbative ladder for the gluelump is equivalent to the BFKL ladder of the pomeron (more exactly to one-half of that ladder cut along the valence gluon line). For the BFKL ladder it was shown 22] that the higher-order correction practically cancels the lowest order result. This fact was used in the glueball case in 11] to neglect the Coulomb ladder completely, and the resulting glueball masses agree surprisingly well with the lattice data (whereas retaining color Coulomb interaction would reduce the lowest masses by $0.5 \mathrm{GeV}$, and increase spin splittings 3 times in strong disagreement with lattice data).

Accordingly for the gluelump case we at first also disregard color Coulomb exchanges both in mass eigenvalues and in wave functions, so that relation (14) holds true and the matrix element $\left\langle\frac{f(r)}{r}\right\rangle$ is to be calculated with eigenfunctions of (7) and (8). One finds that the spacial size of gluelump (in absence of color Coulomb force) is $\sqrt{2}$ times less than that of two-gluon glueballs, and is around $0.5 \mathrm{fm}$ for lowest states. Hence the influence of (however reduced) Coulomb force in this system can be important.

Therefore also calculations have been done for two other values of effective $\alpha_{s}$, which when taking into account higher loop effects 22 reduce to a smaller value, denoted by $\bar{\alpha}_{s}$. Two values of $\bar{\alpha}_{s}, \bar{\alpha}_{s}=0.15$ and $\bar{\alpha}_{s}=0.195$ have 
been used and the results for the spin-overaged mass are shown in Table 2, while the values of spin-orbit matrix elements $b^{\text {(Thomas) }}$ and $b^{(\text {pert })}$ change for $\bar{\alpha}_{s}=0.15$ at most 1.5 times and not listed in the Table.

The resulting values of spin-splitted masses are given in Table 4 for $\bar{\alpha}_{s}=0$.

\section{The gluelump spectrum}

We are now in position to calculate the spectrum of (8) and the spin splittings (11), (12).

The total mass eigenvalue is written as

$$
M\left(J, L, n_{r}\right)=M_{0}(n, L)+\Delta M_{L}+\Delta M_{L S}
$$

where $M_{0}(n, L)$ is the eigenvalue of the operator $h(\mu) \equiv \mu / 2+\mathbf{p}^{2} / 2 \mu+\sigma_{\text {adj }} r$, minimized over the values of $\mu$.

$$
h(\mu) \psi(x)=\varepsilon(\mu) \psi(x) .
$$

Using the standard technic of the QCDSM [3, 6], one finds

$$
\varepsilon(\mu)=\frac{\mu}{2}+\frac{\sigma_{a d j}^{2 / 3} a(n, L)}{(2 \mu)^{1 / 3}}
$$

where $a(n, L)$ are the eigenvalues of the reduced equation found numerically and given in Table 1 (lower entries). Minimization of (17) over $\mu$ yields for $\mu=\mu_{0}$ the values given in Table 1 (upper entries). One has from (17)

$$
\mu_{0}(n, L)=\left(\frac{a(n, L)}{3}\right)^{3 / 4}\left(2 \sigma_{a d j}\right)^{1 / 2} .
$$

From $\varepsilon(\mu)(17)$ one immediately finds that

$$
M_{0}(n, L)=2 \mu_{0}(n, L)
$$

The values of $M_{0}(n, L), \Delta M_{L}$ and the resulting spin-averaged masses $\bar{M}(n, L) \equiv$ $M_{0}(n, L)+\Delta M_{L}$ are given in Table 2 .

Now we turn to the spin splittings of the obtained levels. The values of $\left\langle\frac{1}{r^{3}}\right\rangle$ are taken from (14) and those of $\left\langle\frac{f(r)}{r}\right\rangle$ from the corresponding glueball 
matrix elements in Table 7 of [11], with the proper rescaling from the twogluon Hamiltonian to $h(\mu)$ 11]. The resulting splittings $\Delta M_{L S}$ can be written as

$$
\Delta M_{L S}=\frac{J(J+1)-L(L+1)-2}{2}\left(b^{(\text {Thomas })}(n, L)+b^{(\text {pert })}(n, L)\right)
$$

The values of $b^{\text {(Thomas) }}$ and $b^{(\text {pert })}$ are given in Table 3 . Finally summing up all corrections one obtains the resulting gluelump masses listed in Table 4 for $\bar{\alpha}_{s}=0$.

Now we turn to the two-gluon gluelumps, which correspond to $C=+1$. This is a special case of $3 g$ glueballs when the mass of one gluon tends to infinity. Therefore one can use the same Hamiltonian technic as was exploited for $3 g$ glueballs in [11], i.e. the Hamiltonian $h(\mu)$ in (16) is replaced by $h\left(\mu_{1}, \mu_{2}\right)$ (with $\mu_{i}$ to be found again from minimization of eigenvalues).

$$
h\left(\mu_{1}, \mu_{2}\right)=\frac{\mu_{1}}{2}+\frac{\mu_{2}}{2}+\frac{\mathbf{p}_{1}^{2}}{2 \mu_{1}}+\frac{\mathbf{p}_{2}^{2}}{2 \mu_{2}}+\sigma_{f}\left\{\left|\mathbf{r}_{1}\right|+\left|\mathbf{r}_{2}\right|+\left|\mathbf{r}_{1}-\mathbf{r}_{2}\right|\right\} .
$$

Note the appearence of $\sigma_{f}$ in (21), since the string is now fundamental and forms a triangle, passing through two gluons and the adjoin source. The Hamiltonian (21) can be treated using hyperspherical formalism, for details see Appendix. Here we only quote the result for spin-averaged masses (obtained with accuracy better than $5 \%$ )

$$
M(n, K)=4 \mu_{0}+\omega_{0}\left(n+\frac{1}{2}\right)+\frac{K(K+4)}{15} 4 \mu_{0}
$$

where $n=0,1,2, \ldots ; K=0,1,2, \ldots$ and

$$
\mu_{0}=2 \sqrt{\sigma_{f}}\left(\frac{2}{15}\right)^{1 / 4}(\sqrt{2}+2)^{1 / 2}, \omega_{0}=\frac{4}{\sqrt{5}} \mu_{0} .
$$

For the lowest state, $K=0, n=0$ one obtains

$$
M^{(2 g)}(0,0)=2.61 G e V\left(\sigma_{f}=0.18 G e V^{2}\right) .
$$

The hyperfine splitting of this level into $J^{P C}=0^{++}$and $2^{++}$can be calculated in the same way as in [11] with the result that $0^{++}$lies below $2^{++}$with the interval proportional to $\alpha_{s}$ (see Appendix for explicit expression). We place the spin-averaged value (24) in Table 4 and hyperfine-splitted level 
$0^{++}$in Table 5. It is interesting to note that in two-gluon gluelumps both hyperfine and tensor forces are operating in contrast to one-gluon gluelumps, and therefore the mixing of the state $\mathbf{B}^{(1)} \mathbf{B}^{(2)}$ (which is orbitally excited with respect to $\left.a_{i}^{(1)} a_{i}^{(2)}\right)$ with the latter is significant, which may explain the appearence of this state in lattice calculations.

\section{Discussion of the spectrum. Comparison with lattice data}

The spectrum given in Tables 2 and 4 demonstrates the following features. First of all one can see that the spin splittings of levels are small and can be neglected in first approximation. The ordering of levels has the same character as for two-gluon glueball masses and can be roughly described by the equation

$$
\bar{M}(n, L) \cong(2 n+L) \omega+\bar{M}_{0}
$$

where $\bar{M}_{0}\left(\bar{\alpha}_{s}\right) \cong\left(1.5-2.5 \bar{\alpha}_{s}\right) \mathrm{GeV}$, and $\omega\left(\bar{\alpha}_{s}\right) \approx\left(0.35+\bar{\alpha}_{s} \cdot 1.2\right) \mathrm{GeV}$. This behaviour is typical for other hadrons, see [1] for the discussion of glueballs and [6] for a review of hadronic spectra.

¿From dimensional point of view the spectrum corresponds to the increasing dimension of valence gluon operators $a_{i}, \partial_{i} a_{k}, \partial_{i} \partial_{k} a_{l}$ etc. (and not to the dimension of operators $E_{i}, B_{i}, D_{i} B_{k}$ etc.).

Note that the lowest level, $1^{--}$, is of the electric type and there is a gap of $0.5 \div 0.6 \mathrm{GeV}$ between the ground and excited level, while the distance between $L=0$ and $L=2$ levels is equal to $0.73 \mathrm{GeV}\left(\bar{\alpha}_{s}=0\right)$ or $1 \mathrm{GeV}$ $\left(\bar{\alpha}_{s}=0.15\right)$.

It is instructive to compare our spectrum to the gluelump spectrum of the MIT bag model [23, 24].

For $\Lambda^{1 / 4}=0.315 \mathrm{GeV}$ and $\alpha_{s}=0.23$ the masses obtained in [24] are

$$
\begin{gathered}
M\left(1^{+-}\right)=1.43 \mathrm{GeV}, M\left(2^{--}\right)=1.97 \mathrm{GeV}, M\left(1^{--}\right)=1.98 \mathrm{GeV} \\
M\left(3^{+-}\right)=2.44 \mathrm{GeV}, M\left(2^{+-}\right)=2.64 \mathrm{GeV} .
\end{gathered}
$$

One can see the inverse ordering of the first levels in (26), namely magnetic level $1^{+-}$in MIT bag model is below the electric one $1^{--}$and the gap between them is approximately the same as in QCD string model, but the ordering is reversed. 
Here the difference between two models is most pronounced (in contrast the glueball spectrum, where the lowest two-gluon states have the same ordering in both models, see [11] and [25]). Hence the independent check of lattice calculations can in principle decide which of the models is closer to reality.

Now the recent lattice calculations [26] (see also [1, 2] for earlier lattice studies) yield the mass levels shown in Table 5. One should note at this point, that the (divergent) mass renormalization terms are not subtracted from the data and therefore absolute scale is missing. We therefore have placed for the sake of comparison in Table 5 the lowest $1^{+-}$level at the same mass as given by our calculations, see Table 2 and 4 for $\bar{\alpha}_{s}=0.15$.

Another feature of data from [26] is that only spacial links have been used for gluelump operators at initial and final states, and hence the states in Table 4 containing $E_{j}$ are not directly excited in measurements in [26] (however these states could in principle be excited indirectly due to mixing, i.e. with smaller overlap). The states in Table 4 which are excited directly in [26] are marked by asterix and compared with lattice results in Table 5.

One can see in Table 5 a good correspondence between the levels, and an approximate mass degeneration of $2^{+-}, 3^{+-}$levels is present in both results.

However the $1^{--}, 2^{--}$levels, almost degenerate in the QCDSM have a gap of $0.22 \mathrm{GeV}$ on the lattice. This might be explained by the mixing of the $1^{--}$level with the ground state, having the same quantum numbers, whereas $2^{--}$has no low-lying counterpart.

As was mentioned above, the crucial test of the QCDSM is the search for the ground state level $1^{--}$, which should lie $0.5 \div 0.6 \mathrm{GeV}$ below the lowest $1^{+-}$level found on the lattice. If this level is not found, it would possibly mean that gluelumps are more like bags than strings. However no derivation of the bag model from QCD was ever made and theoretical grounds for this model are of intuitive character, whereas the QCDSM follows directly (with few assumptions supported by lattice data) from the QCD lagrangian. Therefore the existence of the $1^{--}$ground state for the gluelump seems very plausible. In conclusion of this section we discuss shortly an important connection between gluelumps and field strength correlators $D(x), D_{1}(x)$ introduced in [14] and measured on the lattice in [17] using cooling procedure and in [27] using RG smoothing. It is clear from Table 4 that two lowest states, $1^{--}$and $1^{+-}$, correspond to the electric and magnetic correlators respectively, and therefore the masses $M\left(1^{--}\right) \cong 1.12 \mathrm{GeV}$ and $M\left(1^{+-}\right) \cong 1.7$ $\mathrm{GeV}\left(\bar{\alpha}_{s}=0.15\right)$ should give the exponential slopes of those correlators (the 
notation $D_{\|}$and $D_{\perp}$ was also used in [17] coiciding with electric and magnetic respectively). The lowest mass of $M \approx 1 \mathrm{GeV}$ is in agreement with measurements in [17], however for $D_{\perp}$ the same slope was found in contrast with present calculations. There are no general symmetry arguments, why masses (slopes) in both functions should be the same, (in contrast to electric and magnetic condensates at zero temperature) and this disagreement between gluelump masses and slopes in $D_{\|}, D_{\perp}$ calls for further investigation.

One should note in addition, that the independent calculation of the slope from the selfcoupled equations for correlators made in [28], also gives the lowest (electric) mass around $1 \mathrm{GeV}$, in agreement with the present calculations for $\left(\bar{\alpha}_{s}=0.15\right)$.An analysis for the same quantity in the framework of the QCD sum rules 29] gives a larger value in the quenched case, while for the magnetic mass the result was unstable. Finally,in the lattice study [30] of electric and magnetic field correlators made without cooling or smoothing procedures,electric and magnetic masses are obtained in good agreement with present calculations, specifically magnetic mass turned out to be around $0.5 \mathrm{GeV}$ heavier.

\section{Conclusions}

Results of the present calculations in the QCDSM framework are in general agreement with recent lattice data [26], using the restricted set of gluonic operators, and with previous lattice and analytic calculations of the slope of the $D_{\|}$function. It is not clear from the gluelump spectrum point of view why the correlator $D_{\perp}$ (magnetic field correlator) should have the same slope as that of $D_{\|}$, as it was found on the lattice [17. With the lowest gluelump mass equal to $1.2 \div 1.5 \mathrm{GeV}$ in the present calculations, the onset of the adjoint string breaking appears at the string length $r_{0}=1.2 \div 1.5 \mathrm{fm}$, in agreement with recent lattice data 18.

The fundamental role of gluelump states in the theory of the QCD vacuum makes it important to resolve the existing problem of the spectrum and of the relation with vacuum correlators $D_{\perp}, D_{\|}$.

The author is grateful to C.Michael for a useful correspondence, and to Yu.S.Kalashnikova and V.I.Shevchenko for numerous discussions. The present work was partially supported in the framework of the RFFI projects 00-02-17836 and 96-15-96740. This work was completed while the author was visiting the Humboldt University Of Berlin in the framework of the joint 
DFG-RFFI project 96-02-00088G.It is a pleasure for the author to thank M.Mueller- Preussker for a kind hospitality,collaboration and discussions and G.Bali for a series of discussions which stimulated the final version of the paper.

\section{References}

[1] I.Jorysz and C.Michael, Nucl. Phys. B302 (1988) 448.

[2] N.Campbell, I.Jorysz and C.Michael, Phys. Lett. B167 (1986) 91

[3] Yu.A.Simonov, Nucl. Phys. B307 (1988) 512; Phys. Lett. B226 (1989) 151; Yad. Fiz. 54 (1991) 192

[4] A.Yu.Dubin, A.B.Kaidalov, and Yu.A.Simonov, Phys. Lett. B323 (1994) 41; Yad. Fiz. 56 (1993) 213

[5] Yu.A.Simonov, Nucl. Phys. B324 (1989) 67; A.M.Badalian and Yu.A.Simonov, Phys. At. Nucl. 59 (1996) 2164

[6] Yu.A.Simonov, Lectures at the XVII International School of Physics, Lisbon, 1999, hep-ph/9911237

[7] S.I.Kruglov, Phys. Rev. D60 (1999) 116009; hep-ph/9910514

[8] B.O.Kerbikov and Yu.A.Simonov, hep-ph/0001243

[9] A.Yu.Dubin, A.B.Kaidalov, and Yu.A.Simonov, Phys. Lett. B343 (1995) 360; V.L.Morgunov, V.I.Shevchenko and Yu.A.Simonov, Phys. Lett. B416 (1998) 433; Yad. Fiz. 61 (1998) 739

[10] Yu.A.Simonov, JEPT Lett. 57 (1993) 513; A.M.Badalian and Yu.A.Simonov, Phys. At. Nucl. 60 (1997) 630

[11] A.B.Kaidalov and Yu.A.Simonov, Phys. Lett. B (in press), hepph/9911291

[12] C.Morningstar, M.Peardon, hep-lat/991004, G.Bali et al (UKQCD Collaboration), Phys. Lett. B309 (1993) 378, G.Bali, hep-lat/9901023

[13] Yu.A.Simonov, Z. Phys. C53 (1992) 419 
[14] H.G.Dosch, Phys. Lett. B190 (1987) 177, H.G.Dosch and Yu.A.Simonov, Phys. Lett. B205 (1988)339

[15] V.Marquard and H.G.Dosch, Phys. Rev. D35 (1987) 2238

[16] Yu.A.Simonov, Physics-Uspekhi 39 (1996) 313

[17] A.Di Giacomo and H.Panagopoulos, Phys. Lett. B285 (1992) 133, A.Di Giacomo, E.Meggiolaro and H.Panagopoulos, Nucl. Phys. B483 (1997) 371

[18] G.Bali, hep-lat/9908021

[19] V.L.Morgunov, A.V.Nefediev and Yu.A.Simonov, Phys. Lett. B459 (1999) 653

[20] V.I.Shevchenko and Yu.A.Simonov, hep-ph/0001299, Yu.A.Simonov, JETP Lett. (in press), hep-ph/0001244

[21] J.Pantaleone, S.-H.Henry Tye, Y.J.Ng, Phys. Rev. D33 (1986) 777

[22] V.S.Fadin and L.N.Lipatov, Phys. Lett. B429 (1998) 127; M.Ciafaloni and G.Camici, Phys. Lett. B430 (1998) 349; S.Brodsky et al., JETP Lett 70 (1999) 155

[23] M.Chanowitz and S.Sharpe, Phys. Lett. B126 (1983) 225

[24] G.Karl and J.Paton, hep-ph/9904407

[25] J.Kuti, hep-lat/9811021

[26] M.Foster and C.Michael, Phys. Rev. D59 (1999) 094509, heplat/9811010

[27] E.-M.Ilgenfritz and S.Thurner, hep-lat/9905012

[28] Yu.A.Simonov, Phys. At. Nucl. 61 (1998) 855, hep-ph/9712250

[29] M.Eidemueller, H.G.Dosch and M.Jamin, hep-ph/9908318

[30] G.S.Bali, N.Brambilla and A.Vairo, Phys.Lett. B421 (1998) 265

[31] Yu.A.Simonov, Sov. J. Nucl. Phys. 3 (1966) 461; A.M.Badalian and Yu.A.Simonov, Sov. J. Nucl. Phys. 3 (1966) 755; 5 (1967) 60; F.Calogero and Yu.A.Simonov, Phys. Rev. 169 (1968) 789 


\section{Table 1}

Valence gluon masses $\mu_{0}(n, L)$ (upper entries) and reduced eigenvalues $a(n, l)$ (lower entries). $\sigma_{f}=0.18 \mathrm{GeV}^{2}, \sigma_{a d j}=\frac{9}{4} \sigma_{f}=0.405 \mathrm{GeV}$

\begin{tabular}{|l|l|l|l|}
\hline$L \backslash n$ & 0 & 1 & 2 \\
\hline 0 & 0.746 & 1.135 & 1.422 \\
& 2.3381 & 4.0879 & 5.520 \\
\hline 1 & 0.98 & 1.297 & 1.554 \\
& 3.3613 & 4.8845 & 6.216 \\
\hline 2 & 1.168 & 1.443 & \\
& 4.248 & 5.63 & \\
\hline 3 & 1.33 & & \\
& 5.053 & & \\
\hline
\end{tabular}

\section{Table 2}

Unperturbed masses $M_{0}(n, L)$, string correction $\Delta M_{L}$ and spin averaged masses $\bar{M}(n, L)$ for $L=0,1,2,3$ and $n=0$ (upper entries and two last lines) and $n=1$ (lower entries).

\begin{tabular}{|l|l|l|l|l|}
\hline$\backslash L$ & 0 & 1 & 2 & 3 \\
\hline$M_{0}$ & 1.492 & 1.96 & 2.336 & 2.66 \\
$(\mathrm{GeV})$ & 2.27 & 2.6 & 2.886 & \\
\hline$\Delta M_{L}$ & 0 & -0.0581 & -0.103 & -0.139 \\
$(\mathrm{GeV})$ & 0 & -0.025 & -0.0546 & \\
\hline$M$ & 1.492 & 1.9 & 2.233 & 2.52 \\
$(\mathrm{GeV})$ & 2.27 & 2.575 & 2.83 & \\
\hline$M\left(\bar{\alpha}_{s}=0.15\right)$ & 1.12 & 1.73 & 2.15 & 2.46 \\
\hline$M\left(\bar{\alpha}_{s}=0.195\right)$ & 0.982 & 1.67 & 2.10 & \\
\hline
\end{tabular}


Table 3

Perturbative and Thomas spin-orbit martix elements defined as in (11 13)

\begin{tabular}{|l|l|l|l|}
\hline$L$ & 1 & 2 & 3 \\
\hline$b^{(\text {pert })}$ & 0.033 & 0.0096 & 0.00425 \\
\hline$b^{(\text {Thomas })}$ & -0.0025 & -0.0024 & -0.0021 \\
\hline
\end{tabular}

\section{Table 4}

Gluelump masses for lowest states $J^{P C}$ are listed together with gluonic state operators in the background field formalism and in the general notations. Asterix marks the states which are directly generated on the lattice 26

\begin{tabular}{|l|c|c|c|c|l|}
\hline State & \multirow{2}{*}{$L$} & $n$ & \multicolumn{2}{|c|}{ operator } & $\begin{array}{l}\text { Mass } \\
(\mathrm{GeV})\end{array}$ \\
\cline { 4 - 5 } & & & backgr. & general & 1.492 \\
$1^{--}$ & 0 & 0 & $a_{i}$ & $E_{i}$ & $1.87^{*}$ \\
$1^{+-}$ & 1 & 0 & $e_{i k l} \partial_{k} a_{l}$ & $B_{i}$ & 1.93 \\
$2^{+-}$ & 1 & 0 & $\left(\partial_{i} a_{k}\right)_{s y m m}$ & $\left(D_{i} E_{k}\right)_{s y m m}$ & 2.27 \\
$1^{--}$ & 0 & 1 & $a_{i}$ & $E_{i}$ & $2.21^{*}$ \\
$1^{--}$ & 2 & 0 & $\left(\partial_{i} \partial_{k} a_{l}\right)_{J}$ & $D_{i} B_{k} e_{i k l}$ & $2.226^{*}$ \\
$2^{--}$ & 2 & 0 & $"$ & $\left(D_{i} B_{k}\right)_{s y m m}$ & 2.24 \\
$3^{--}$ & 2 & 0 & $"$ & $D_{i} D_{k} E_{l}$ & $2.51^{*}$ \\
$2^{+-}$ & 3 & 0 & $\left(\partial_{i} \partial_{k} \partial_{l} a_{m}\right)_{J}$ & $\left(D_{i} D_{k} B_{l}\right)_{J}$ & $2.52^{*}$ \\
$3^{+-}$ & 3 & 0 & $"$ & $"$ & 2.53 \\
$4^{+-}$ & 3 & 0 & $"$ & $\left(D_{i} D_{k} D_{l} E_{m}\right)_{4}$ & $2.57^{*}$ \\
$1^{+-}$ & 1 & 1 & $\left(\partial_{i} a_{k}\right)_{1}$ & $B_{i}$ & 2.57 \\
$2^{+-}$ & 1 & 1 & $\left(\partial_{i} a_{k}\right)_{2}$ & $D_{i} E_{k}$ & $2.61^{*}$ \\
$0^{++} 2^{++}$ & 0 & 0 & $\left(a_{i}^{(1)} a_{k}^{(2)}\right)_{J}$ & $\left(\mathbf{B}_{i}^{(1)} \mathbf{B}_{k}^{(2)}+\mathbf{E}_{i}^{(1)} \mathbf{E}_{k}^{(2)}\right)_{J}$ & 2 \\
\hline
\end{tabular}




\section{Table 5}

The gluelump states generated by spacial operators, with masses computed analytically in the present paper $\left(\bar{\alpha}_{s}=0.15\right)$ and masses computed on the lattice [26]. For better comparison results of Table 2 corrected for larger $\sigma_{f}=0.22$ used on lattice.

\begin{tabular}{|l|l|l|l|l|l|l|}
\hline$J^{P C}$ & $1^{+-}$ & $1^{--}$ & $2^{--}$ & $2^{+-}$ & $3^{+-}$ & $0^{++}$ \\
\hline $\begin{array}{l}M(\mathrm{GeV}) \\
\text { lattice }\end{array}$ & 1.87 & 2.23 & 2.45 & 2.84 & 2.84 & 2.96 \\
\hline $\begin{array}{l}M(\mathrm{GeV}) \\
\text { QCD string }\end{array}$ & 1.87 & 2.34 & 2.36 & 2.70 & 2.71 & 2.78 \\
\hline
\end{tabular}




\section{Appendix}

\section{Two-gluon gluelumps}

Consider a static source at point $\mathbf{r}_{3}=0$ and two gluons at $\mathbf{r}=\mathbf{r}_{1}$ and $\mathbf{r}=\mathbf{r}_{2}$, connected by a fundamental string, passing through these three points. The Hamiltonian for the case, when both orbital momenta are zero (no string rotation correction) can be written in analogy to (7) as

$$
h\left(\mu_{1}, \mu_{2}\right)=\frac{\mu_{1}+\mu_{2}}{2}+\frac{\mathbf{p}_{1}^{2}}{2 \mu_{1}}+\frac{\mathbf{p}_{2}^{2}}{2 \mu_{2}}+\sigma_{f}\left\{\left|\mathbf{r}_{1}\right|+\left|\mathbf{r}_{2}\right|+\left|\mathbf{r}_{1}-\mathbf{r}_{2}\right|\right\}
$$

This 3 body problem can be treated by the hyperspherical formalism [31. Introducing the hyperradius $\rho$ and denoting $\mu_{1}=\mu_{2}=\mu$, one has

$$
\rho^{2}=\boldsymbol{\eta}^{2}+\boldsymbol{\xi}^{2}, \quad \boldsymbol{\eta}=\mathbf{r}_{12} / \sqrt{2}, \quad \boldsymbol{\xi}=\left(\mathbf{r}_{1}+\mathbf{r}_{2}\right) \frac{1}{\sqrt{2}}
$$

one obtains the Hamiltonian for the given grand orbital momentum $K=$ $0,1,2, \ldots$

$$
h=+\mu-\frac{1}{2 \mu \rho^{5}} \frac{\partial}{\partial \rho}\left(\rho^{5} \frac{\partial}{\partial \rho}\right)+\frac{\left(K+\frac{3}{2}\right)\left(K+\frac{5}{2}\right)}{2 \mu \rho^{2}}+U_{K}(\rho)
$$

where $U_{K}(\rho)$ for $K=0$ is the potential term in (A.1) averaged over all hyperangles. Using standard expressions from [31] one obtains

$$
U_{0}(\rho)=C_{0} \sigma \rho, C_{0}=\frac{32 \sqrt{2}(1+\sqrt{2})}{15 \pi}
$$

Eq. (A.3) can be solved numerically and results are tabulated, but it appears that the accuracy of about $1 \%$ for the eigenvalue can be obtained by the stationary point method applied to the effective potential $W(\rho)$

$$
W(\rho)=\frac{\left(K+\frac{3}{2}\right)\left(K+\frac{5}{2}\right)}{2 \mu \rho^{2}}+U_{K}(\rho) ; \quad W^{\prime}\left(\rho=\rho_{0}\right)=0 ;
$$

while the second derivative at $\rho=\rho_{0}$ defines the radial excitation energy $\omega$ :

$$
W^{\prime \prime}\left(\rho_{0}\right) \frac{\left(\rho-\rho_{0}\right)^{2}}{2} \equiv \frac{\mu \omega^{2}\left(\rho-\rho_{0}\right)^{2}}{2}
$$


In this way one obtains the mass eigenvalue $M(\mu)$, and finally the stationary point condition for $\mu, \frac{d M}{d \mu}\left(\mu=\mu_{0}\right)=0$ defines gluon constituent mass $\mu_{0}$ and the resulting mass eigenvalue $M\left(\mu_{0}\right)$, given in (22)-(24). One can note that radial excitations are given by $\omega_{0}=0.955 \mathrm{GeV}$, and "grand-orbital excitation" from $K=0$ to $K=1$ is given by the interval $\Delta M=0.712 \mathrm{GeV}$.

The hyperfine splitting is given by the Hamiltonian (cf the discussion in [11] for $3 g$ glueballs)

$$
H_{S S}=\mathbf{S}^{(1)} \mathbf{S}^{(2)} \frac{5 \pi C_{2}(\text { fund }) \alpha_{s}}{3 \mu_{0}^{2}}\left\langle\delta^{(3)}\left(\mathbf{r}_{12}\right)\right\rangle_{\rho}
$$

The last factor in (A.7) is easily computed

$$
\left\langle\delta^{(3)}\left(\mathbf{r}_{12}\right)\right\rangle_{\rho}=\frac{\sqrt{2}}{\pi^{2} \rho_{0}^{3}}, \quad \rho_{0}=1.15 / \mu_{0} .
$$

This yields the spin splitting of the levels

$$
\Delta M_{S S}=\mathbf{S}^{(1)} \mathbf{S}^{(2)} 0.49 \mu_{0} \frac{4}{3} \alpha_{s}
$$

For $\alpha_{s}=\bar{\alpha}_{s}=0.15$ one obtains

$$
\Delta M_{S S}=0.0525 \mathrm{GeV}\left(\begin{array}{ll}
-2, & J=0 \\
+1, & J=2
\end{array}\right) .
$$

In this way one gets the mass $M\left(0^{++}\right)$in Table 5 . 